\title{
Contamination, a Major Problem in Nuclear Medicine Imaging: How to Investigate, Handle, and Avoid It
}

\author{
Narvesh Kumar ${ }^{1}$, Shashwat Verma ${ }^{1}$, Rani Kunti R. Singh ${ }^{2}$, Deepanksha Datta ${ }^{1}$, Subhash Chandra Kheruka ${ }^{1}$, \\ and Sanjay Gambhir ${ }^{1}$ \\ ${ }^{I}$ Department of Nuclear Medicine, SGPGIMS, Lucknow, Uttar Pradesh, India; and ${ }^{2}$ Department of Radiodiagnosis, Vivekananda \\ Polyclinic and Institute of Medical Sciences, Lucknow, Uttar Pradesh, India
}

\begin{abstract}
We present a case study in which artifacts from collimator contamination and patient motion were seen on a bone scan. Any identified artifact must be further investigated and documented so as to detect its source and thus prevent its future occurrence.
\end{abstract}

Key Words: collimator contamination; ${ }^{99 m T c-M D P ; ~ m o t i o n ~ a r t i f a c t ; ~}$ bone scan

J Nucl Med Technol 2017; 45:241-242

DOI: 10.2967/jnmt.117.190256

\section{$\mathbf{R}$} adioactive contamination and image artifacts can be introduced at any time during a nuclear scan. Contamination leads to unwanted radiation exposure to patients, caregivers, and staff, as well as interrupting the acquisition schedule (1).

We report a case of collimator contamination and motion artifact that occurred during a bone scan. Our purpose is to describe the pattern of this artifact so as to help nuclear medicine technologists and physicians identify it.

\section{CASE STUDY}

A 55-y-old man with a known case of prostate carcinoma underwent whole-body bone scanning to check for skeletal metastases. A $740-\mathrm{MBq}$ injection of ${ }^{99 \mathrm{~m}} \mathrm{Tc}-$ methylene diphosphonate was given intravenously, and delayed whole-body anterior and posterior planar images from the skull to the feet were acquired on a dual-head SPECT system (Hawkeye 4; GE Healthcare).

The images revealed 3 abnormal foci of radiotracer outside the body contour near the right foot, predominantly in the posterior projection. Well-defined linear tracer activity was also noted arising from these extracorporeal tracer foci, most prominently in the center of body but also

\footnotetext{
Received Jan. 24, 2017; revision accepted Apr. 1, 2017.

For correspondence or reprints contact: Narvesh Kumar, Department of Nuclear Medicine, SGPGIMS, Raebareli Rd., Lucknow-226014, Uttar Pradesh, India.

E-mail: kumarnarvesh@gmail.com

Published online Apr. 13, 2017.

COPYRIGHT (c) 2017 by the Society of Nuclear Medicine and Molecular Imaging.
}

faintly at the medial aspect of the right thigh. In addition, there was blurring of the head-and-neck region on both anterior and posterior images because the patient had moved during the acquisition (Fig. 1).

A spot image of the head, neck, and thorax was acquired with the patient in head-first supine position to correct the motion artifact. As we changed the orientation of the patient from feet-first supine to head-first supine, the foci of abnormal update that had been seen outside the body contour near the right foot were now seen in the right upper thoracic region around the first and second ribs. Though false-positive, this finding might be reported as metastatic disease if not scrutinized adequately (Fig. 2).

The patient was then removed from the camera and images of the collimators alone were acquired, with the two collimators facing each other. The bright foci of tracer were well evident on these images (posterior $>$ anterior) and hence were determined to be due to contamination of the collimators (Fig. 3).

\section{DISCUSSION}

Contamination can be categorized as either directly patient-related, such as contamination of the clothing or hair, or indirectly patient-related, such as contamination of the patient's surroundings (camera detectors, floors, sink, dustbins). Patient contamination can be identified easily during image acquisition, but because surrounding

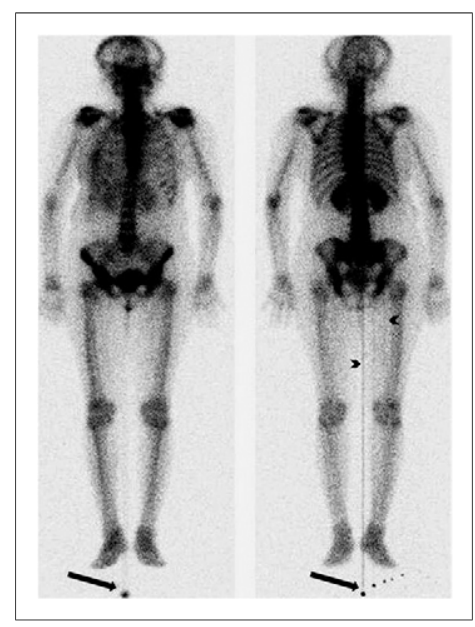

FIGURE 1. Anterior (left) and posterior (right) whole-body bone scans revealing contamination foci (arrows) from which arises well-defined linear activity, most prominently in center of body and also faintly at medial aspect of right thigh (arrowheads). Motion artifact in headand-neck region is also seen. 


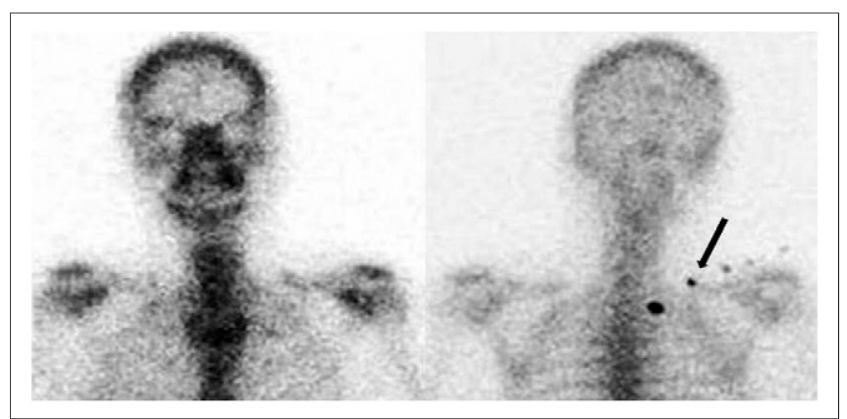

FIGURE 2. With patient in head-first supine position, there is no motion artifact in spot static anterior (left) or posterior (right) images of head, neck, and thorax, but contamination that was seen beside right foot previously is now seen in right upper thoracic region (arrow).

contamination cannot be so easily identified, daily wipe tests are mandatory (2).

Interpretation of a nuclear scan can be affected by many kinds of technical errors during its acquisition, as well as by injection artifacts, prosthetic implants, patient motion, and extracorporeal contamination such as that of the patient's clothes, the table, or the collimator (I). The possibility of false-positive results due to technical errors or contamination should always be kept in mind, especially if the lesions are prominent in a single projection (3).

Contamination of the detector or collimator usually involves a limited area and appears as a line throughout whole-body images (3). Detector contamination differs from direct patient contamination; in the latter, the location of the radiotracer changes with movement of the patient, whereas in the former, the location remains static. In our case, the observed contamination of the collimators was suspected to be due to urinary spillage during the direct radionuclide cystogram that had been performed just before the bone scan.

Patient motion is also a common source of artifacts in images but usually can easily be detected by cine displays, sinograms, and summed planar images. To avoid any discomfort-induced motion artifacts, the technologist should ensure that the patient is comfortably positioned during the image acquisition. If needed, an arm support, holding straps, and sandbags may also be used to reduce patient movement during the acquisition (4).

Care should be taken to avoid contamination and other artifacts, as they interrupt the workflow of nuclear medicine
FIGURE 3. Anterior (top) and posterior (bottom) images obtained without patient and table reveal multiple foci of contamination (arrows) on collimators, more prominent in posterior image.

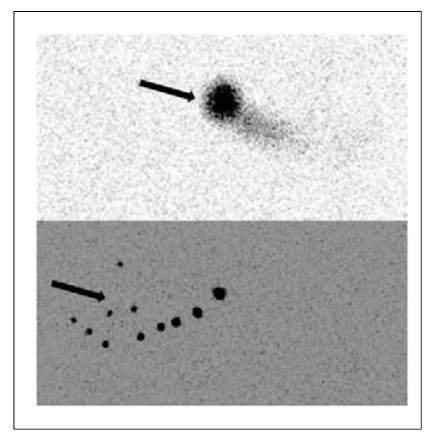

technologists and physicians (because of the time needed for cleaning and decontamination) and expose patients to additional radiation (because of the need to repeat the imaging study).

\section{CONCLUSION}

Nuclear medicine technologists-usually being the first to encounter an instance of a potential artifactmust have a thorough knowledge of such problems and the experience to identify them. Nuclear medicine physicians also need to have a sound knowledge of such sources of error and the ability to differentiate a falsepositive finding from a true pathologic abnormality. Proper training programs and designation of duties is of the utmost importance for the personnel in a nuclear medicine facility to avoid and manage radioactivity-related accidents such as artifacts. Any identified artifact must be investigated as to its source and documented to prevent its future occurrence.

\section{DISCLOSURE}

No potential conflict of interest relevant to this article was reported.

\section{REFERENCES}

1. Mosman EA, Peterson LJ, Hung JC, Gibbons RJ. Practical method for reducing radioactive contamination incidents in the nuclear cardiology laboratory. $\mathrm{J} \mathrm{Nucl}$ Med Technol. 1999;27:287-289.

2. Kasner DL, Spieth ME. The day of contamination. J Nucl Med Technol. 2003;31:21-24.

3. Assadi M, Ebrahimi A, Eftekhari M, et al. A simple way to distinguish bed clothing contamination in a whole body bone scan: a case report. J Med Case Reports. 2007;1:173.

4. Naddaf SY, Collier BD, Elgazzar AH, Khalil MM. Technical errors in planar bone scanning. J Nucl Med Technol. 2004;32:148-153. 